\title{
Análise comparativa de estratégias qualitativas de investigação: possibilidades para a pesquisa em turismo
}

\section{Comparative analysis of qualitative investigation strategies: possibilities for research in tourism}

\author{
Márcia Shizue Massukado (MASSUKADO, M. S.) ${ }^{*}$
}

\begin{abstract}
RESUMO: As estratégias de investigação nas abordagens qualitativa, quantitativa ou multi-métodos auxiliam a exploração da realidade e o entendimento de fenômenos sociais permitindo que as perspectivas teóricas sejam testadas e/ou analisadas na prática. Nesse contexto, o artigo pretende apresentar comparativamente as principais estratégias de pesquisa qualitativa, ressaltando suas possibilidades para os estudos em turismo. $\mathrm{O}$ texto parte de uma visão geral da pesquisa, enumerando suas abordagens $\mathrm{e}$ aprofundando as características da pesquisa qualitativa, além de contemplar uma síntese de cada técnica. Ao final uma discussão de caráter sugestivo apresenta a importância da triangulação como forma de se trabalhar com múltiplas técnicas e enriquecer a confiabilidade e validade dos dados coletados independentemente da estratégia de pesquisa escolhida.
\end{abstract}

Palavras-chave: metodologia de pesquisa; estratégias qualitativas de investigação; pesquisa em turismo.

ABSTRACT: Investigation strategies in qualitative, quantitative, or multi-method approaches assist in exploring the reality and the understanding of social phenomena and, thus, allow theoretical perspectives to be tested and/or analyzed in practice. In this context, the article intends to present, comparatively, the main strategies involved in qualitative research, highlighting its possibilities for tourism studies. The text begins with an overview of the research project, listing its approaches, deepening the characteristics of qualitative research, and presenting a summary of each technique to be analyzed. In the end, a suggestive discussion presents the importance of triangulation as a way to work with multiple techniques and enrich the validity of the data that are collected, regardless of the selected research strategy.

Keywords: research methodology; qualitative research strategies; tourism research.

\footnotetext{
* Bacharel em Turismo pela Universidade Federal do Paraná - UFPR. Mestre em Administração - UFPR e Doutoranda em Administração pelo CEPPAD-UFPR. Professora do Departamento de Turismo da UFPR.E-mail:massukado@ufpr.br
} 


\section{INTRODUÇÃO}

As pesquisas desenvolvidas nas mais diversas áreas de conhecimento sejam elas relacionadas às ciências sociais e humanas ou às ciências exatas ou biológicas, necessitam de regras e processos que delimitem, espaciotemporalmente, o objeto de estudo: o método científico.

As possibilidades de desenvolvimento da pesquisa enfatizam a variabilidade dos métodos e abordagens existentes ou em desenvolvimento, pois a realidade sob uma epistemologia interpretativista, na visão de Grix (2002) ou posição hermenêutica de Marsh e Furlong (2002) é complexa e construída socialmente, indicando que a evolução da sociedade desencadeia descobrimentos e novas formas de ver o mundo, necessitando inovar também as medidas para mensurar seus fenômenos.

Entretanto os procedimentos de pesquisa podem variar independentemente do fenômeno ou problemática adotada no estudo. Os estudos sobre redes em turismo exemplificam essa prática. Embora eminentemente de caráter quantitativo, a pesquisa sobre redes e relacionamentos na atividade turística tem demonstrado certa preocupação em utilizar-se de métodos qualitativos, como as pesquisas realizadas por Novelli et al (2006) e Saxena (2005). Lynch (2000), em seu estudo sobre redes no setor de hospitalidade, utiliza-se de ambos os métodos de pesquisa, evidenciando primeiramente técnicas qualitativas, seguidas de análises estatísticas. De certa forma, pode-se considerar que o confinamento a métodos exclusivamente quantitativos tende a reduzir o caráter analítico e explicativo de uma pesquisa.

Em face deste contexto, a infinidade de abordagens metodológicas de pesquisa permite que o pesquisador observe e estude seu objeto por várias lentes e diferentes ângulos de visão, enriquecendo seu estudo e a confiabilidade dos resultados auferidos.

Ao considerar tais assertivas, este artigo busca analisar comparativamente as diversas abordagens em métodos qualitativos de pesquisa, apresentando possibilidades práticas para os estudos no setor de turismo. Nesse sentido, este trabalho procurou relatar pesquisas teóricas e teórico-empíricas que apresentam as principais técnicas de pesquisa qualitativa, utilizadas em estudos organizacionais, construindo um quadro 
comparativo que confrontasse as características de cada método, suas aplicações (campos e finalidades), os principais autores, os pontos fortes e os desafios (preconceitos e limitações) de aplicação dessas técnicas.

\section{A ABORDAGEM QUALITATIVA NAS PESQUISAS: DEFINIÇÕES, CARACTERÍSTICAS E APLICAÇÕES NA PESQUISA EM TURISMO}

O delineamento de uma pesquisa requer conhecimento sobre qual a melhor forma de se descobrir, por meios científicos, a resposta para uma problemática estabelecida. Essa melhor forma pode emergir por diversos fatores: interesse e habilidade do pesquisador para conduzi-la, tempo e recursos disponíveis, enquadramento do método ao objeto de pesquisa e principalmente pelos pressupostos ontológicos e epistemológicos adotados pelo pesquisador.

Conforme Creswell (2003, p. 18), “os pressupostos (ou paradigmas), as estratégias e o método contribuem como um todo para uma abordagem de pesquisa quantitativa, qualitativa ou multi-método". Para o autor a importância dessas três abordagens recai sobre a existência de um crescente interesse no uso da pesquisa qualitativa, uma emergência nas abordagens multi-método e um contínuo uso das formas tradicionais de delineamento quantitativo. A distinção das abordagens é descrita da seguinte forma:

Uma abordagem quantitativa é a que o investigador primeiramente utiliza os pressupostos pós-positivistas para o desenvolvimento do conhecimento (...), emprega estratégias como experimentos e levantamentos e coleta dados por instrumentos pré-determinados que resultem em dados estatísticos. Alternativamente, a abordagem qualitativa (...) baseia-se em perspectivas construtivistas ou participativas. Utiliza estratégias de pesquisa como narrativas, fenomenologias, etnografias, estudos de grounded theory ou estudos de caso. $\mathrm{O}$ pesquisador coleta dados não estruturados e emergentes (...). Finalmente, a abordagem de multi-método é aquela em que o pesquisador tende a basear seus pressupostos em campos pragmáticos. Emprega estratégias que envolvem a coleta de dados tanto simultaneamente ou sequencialmente para melhor entender os problemas de pesquisa. A coleta de dados envolve tanto informações numéricas quanto informações textuais (CRESWELL, 2003, p. 19-20, grifo nosso). 
De maneira geral, a maioria dos autores faz a distinção entre as abordagens somente em relação a pesquisas qualitativas e quantitativas; entretanto Bryman (1984) releva que tais métodos podem também ser complementares, corroborando a idéia de Punch (1998), ao destacar a crescente tendência de combinação de ambos, além de Patton (2002) reafirmar a questão de que essas abordagens não são mutuamente excludentes.

O entendimento do conceito de pesquisa qualitativa ou abordagem qualitativa de pesquisa é variado no campo das ciências. Denzin e Lincoln (2006) argumentam que a pesquisa qualitativa é, em si mesma, um campo de investigação, envolve o estudo do uso e a coleta de uma variedade de materiais empíricos e, como um conjunto de atividades interpretativas, não privilegia nenhuma única prática metodológica em relação a outra. Diante dessas características, as autoras definem genericamente e de maneira inicial que "a pesquisa qualitativa é uma atividade situada que localiza o observador no mundo" (DENZIN e LINCOLN, 2006, p. 17).

Miles e Huberman (1994) não apresentam uma definição rígida do termo pesquisa qualitativa, mas destacam algumas características recorrentes da pesquisa naturalística, encontradas nessa abordagem: contato prolongado com o campo ou a situação real, o papel do pesquisador em garantir uma visão holística do fenômeno e capturar os dados por meio das percepções dos atores locais, suspendendo seus preconceitos sobre os tópicos pesquisados. Ainda segundo os autores muitas interpretações do material são possíveis, relativamente pouca padronização instrumental é utilizada e a maioria das análises é feita utilizando-se palavras.

Na visão de Creswell (1998), similarmente ao apresentado por Denzin e Lincoln (2006), mas com menor foco para as fontes de informação, a pesquisa qualitativa pode ser definida como:

Um processo de investigação e entendimento baseado em tradições de investigação metodológicas que exploram o problema humano e social. $\mathrm{O}$ pesquisador constrói um quadro complexo e holístico, analisa palavras, reporta detalhadamente as visões de informantes e conduz o estudo em um campo natural (CRESWELL, 1998, p. 15) 
Outra forma de conceituar a pesquisa qualitativa pode dar-se por intermédio da definição dos tipos de dados ou informação que são classificados como qualitativos. Patton (2002) considera nesse rol de dados as entrevistas com citações verbalizadas para serem interpretadas, as observações e descrições de campo detalhadas que incluem o contexto dessas observações e os documentos ou informações selecionadas de documentos que gravem e preservem o contexto.

O destaque para as inúmeras definições existentes para a abordagem qualitativa dos estudos revela também variadas estratégias de investigação e métodos de pesquisa que podem ser aplicados com o intuito de verificar o significado de um fenômeno em seu ambiente natural. Algumas características da pesquisa qualitativa, elencadas na tabela 01 , merecem destaque, pois auxiliam o pesquisador em sua escolha e no estabelecimento dos parâmetros de seu estudo.

\section{TABELA 01 - CARACTERÍSTICAS DA PESQUISA QUALITATIVA}

\begin{tabular}{l|l}
\hline \multicolumn{1}{c|}{ Autor } & \multicolumn{1}{c}{ Características } \\
\hline Bryman (1984) & Compromisso em ver o mundo social do ponto de vista do ator \\
\hline $\begin{array}{l}\text { Denzin e Lincoln } \\
(2006)\end{array}$ & Énfase sobre as qualidades das entidades e sobre processos e os significados \\
\hline $\begin{array}{l}\text { Cassell e Simon } \\
(1994)\end{array}$ & $\begin{array}{l}\text { Permite que o pesquisador, com o avanço de sua pesquisa, altere a natureza de } \\
\text { sua intervenção em resposta à natureza mutante do contexto }\end{array}$ \\
\hline $\begin{array}{l}\text { Wolcott (1975) })^{1} \text { apud } \\
\text { Borman et al (1986) }\end{array}$ & O pesquisador é notadamente o instrumento de pesquisa. \\
\hline Patton (2002) & Os dados tipicamente eclodem durante a ida a campo \\
\hline Silverman (2000) & $\begin{array}{l}\text { Os métodos utilizados exemplificam a crença de que eles podem sustentar um } \\
\text { entendimento mais profundo do fenômeno social que os métodos quantitativos }\end{array}$ \\
\hline Creswell (2003) & $\begin{array}{l}\text { É fundamentalmente interpretativo o que permite que o pesquisador conduza a } \\
\text { interpretação dos dados }\end{array}$ \\
\hline $\begin{array}{l}\text { Miles e Huberman } \\
\text { (1994) }\end{array}$ & $\begin{array}{l}\text { A narrativa não possui formatos fixos, deve combinar elegância teórica com uma } \\
\text { descrição credível do objeto }\end{array}$ \\
\hline Patton (2002) & A confiabilidade recai sobre a competência, habilidade e o rigor do pesquisador \\
\hline Creswell (2003) & $\begin{array}{l}\text { A validade é utilizada para sugerir estabelecendo se as descobertas estão em } \\
\text { conformidade com o ponto de vista do pesquisador, do participante ou dos } \\
\text { leitores }\end{array}$ \\
\hline
\end{tabular}

FONTE: Elaborado pela autora com base em Bryman (1984), Denzin e Lincoln (2006), Cassell e Simon (1994), Wolcott (1975) apud Borman et al (1986), Patton (2002), Silverman (2000), Creswell (2003), Miles e Huberman (1994), Patton (2002).

1 WOLCOTT, H. F. Criteria for an Ethnographic Approach to Research in Schools. Human Organization, 34, 2, 111-27, 1975 
O processo de pesquisa na abordagem qualitativa contempla, geralmente, etapas bem definidas. Segundo Denzin e Lincoln (2006), a distinção pode ser feita em cinco fases, abordando o pesquisador, as perspectivas teóricas, estratégias de pesquisa, métodos de coleta e análise e a interpretação e apresentação.

De outra forma, Saunders et al (2000) argumentam que existe a necessidade de se tratar do processo de pesquisa como uma 'cebola', em que cada uma das camadas faz emergir uma questão e uma decisão sobre a linha metodológica que se pretende seguir. Para os autores o processo segue as seguintes 'camadas': filosofia da pesquisa, enfoque da pesquisa, estratégia da pesquisa, horizonte de tempo e método de coleta de dados.

A análise do processo de pesquisa apresentado na literatura aponta sutis diferenças em estruturar e apresentar a pesquisa qualitativa. Contudo todos os autores destacam a necessidade de um instrumento para coletar os dados e conduzir a pesquisa em campo, sob as mais diversas denominações: estratégia de investigação (CRESWELL, 2003), estratégia de pesquisa (DENZIN e LINCOLN, 2006; SAUNDERS et al, 2000), estratégias de campo ou frameworks qualitativos (PATTON, 2002), métodos de pesquisa qualitativa (SILVERMAN, 2000), tradição de investigação (CRESWELL, 1998), métodos analíticos (MILES e HUBERMAN, 1994), entre outras nomenclaturas.

\section{ESTRATÉgIAS DE INVESTIGAÇÃo EMPREgADAS NA PESQUISA QUALITATIVA}

As estratégias de investigação representam a forma como o pesquisador irá intervir em campo para coletar dados e informações necessárias à sua pesquisa. Creswell (1998) em sua análise sobre a investigação qualitativa, propõe cinco tradições: a biografia, a fenomenologia, os estudos em grounded theory, a etnografia e os estudos de caso. Denzin e Lincoln (2006) apresentam como estratégias de pesquisa o estudo de caso, etnografia, observação participante, etnografia da performance, fenomenologia, 
etnometodologia, grounded theory, história de vida, método histórico, pesquisa ação e pesquisa aplicada, pesquisa clínica.

Apesar de essas propostas estabelecerem diferentes estratégias de investigação, que fazem parte da pesquisa qualitativa, Patton (2002, p. 13) oferece uma forma simples de escolha entre métodos quantitativos e qualitativos ao exemplificar, de maneira pragmática: "se você quer saber quanto uma pessoa pesa, use uma escala (...). Se você quer entender o que o peso significa para ela (...) você precisa questioná-la, descobrir suas experiências e ouvir suas histórias".

Baseada nessa assertiva de busca pelo significado e exploração de problemas sociais - objetivos constantemente recorrentes nos estudos turísticos -, as seguintes estratégias de pesquisa serão analisadas comparativamente: estudo de caso, etnografia, fenomenologia, grounded theory, pesquisa-ação, observação participante, história de vida.

\section{Estudo de caso}

De acordo com Eisenhardt (1989, p. 534) “o estudo de caso é uma estratégia de pesquisa que foca no entendimento da dinâmica presente em um determinado local'. A existência de múltiplas fontes de evidência no estudo de caso se deve, segundo a autora, à combinação de métodos de coleta de dados, como arquivos, entrevistas, questionários e observações.

Yin (2001, p. 32) desenvolve sua definição para estudo de casos, apresentando primeiramente o escopo de um estudo de caso: "é uma investigação empírica que investiga um fenômeno contemporâneo dentro de seu contexto da vida real, especialmente quando os limites entre o fenômeno e o contexto não estão claramente definidos".

Outras características, como a coleta de dados e as estratégias de análise de dados são relevadas posteriormente: "a investigação de estudo de caso enfrenta uma situação tecnicamente única (...) e, como resultado, baseia-se em várias fontes de evidências, (...) e beneficia-se do desenvolvimento prévio de proposições teóricas para conduzir a coleta e a análise de dados" (YIN, 2001, p. 32-33). 
Creswell (1998) destaca algumas características do estudo de caso. Segundo o autor um estudo de caso é a exploração de um 'sistema fechado' ou um caso/múltiplos casos, com fronteiras que definem tempo e espaço, utilizando-se de múltiplas fontes de informação, envolvendo um contexto social, físico, histórico e/ou econômico, podendo ser um estudo intrínseco (pela sua unicidade) ou instrumental (ilustrativo).

Especificamente na pesquisa em turismo, Clark et al (1998) citam como exemplo de aplicação deste método, um estudo de caso sobre as reações dos consumidores a um novo conceito de parque temático. Por outro lado, os autores também exemplificam um estudo de caso múltiplo que tratasse da comparação entre a experiência dos visitantes em diferentes parques temáticos.

\section{Etnografia}

É sabido que a etnografia tem suas raízes nos estudos antropológicos, migrando vagarosamente para a sociologia e os estudos organizacionais, principalmente sob o enfoque da observação participante, apesar de ambas as estratégias de pesquisa terem objetivos distintos. De acordo com Sanday (1979) a utilização da observação participante em estudos etnográficos é suplementada por uma variedade de instrumentos de coleta de dados, como: entrevistas com informantes-chave, coleta de história de vida, questionários e entrevistas estruturadas.

Creswell (1998) define que etnografia é a descrição e interpretação de um grupo cultural e/ou social ou um sistema. Analisando estudos sociológicos, o autor afirma que o pesquisador inicia seu estudo examinando as pessoas e suas interações em locais comuns e tentando discernir padrões presentes como ciclos de vida, eventos e assuntos culturais.

De uma maneira menos convencional, Hammersley e Atkinson (1995) definem o termo etnografia como método particular ou conjunto de métodos, que requer um pesquisador participante - disfarçado ou não - no dia-a-dia das pessoas por um extenso período de tempo, observando o que acontece, ouvindo o que é dito e questionando, quando necessário. 
As dificuldades operacionais deste método de pesquisa, de acordo com Van Maanen (1979), relacionam-se tanto com a confusão que circunda os vários tipos de informações empíricas geradas pelo estudo etnográfico quanto pela confusão dos usos teóricos dessa informação.

Clark et al (1998) suscitam ainda que a etnografia tem como pontos problemáticos a questão do acesso ao local escolhido para realização da pesquisa e o tempo que é consumido na condução da pesquisa.

\section{Fenomenologia}

Os trabalhos de Edmund Husserl (1859-1938) ${ }^{2}$ são um marco no estudo da fenomenologia, segundo os quais o objetivo da fenomenologia é estudar como os fenômenos humanos são experenciados na consciência, em atos perceptíveis e cognitivos, bem como 'analisar' como eles podem ser valorados e apreciados esteticamente.

Creswell (1998) complementa que tal abordagem inclui o estudo de problemas relacionados a adentrar o campo de percepção dos participantes, vendo como eles experienciam, vivem e expõem o fenômeno, à procura do significado das experiências para os participantes.

De acordo com Merleau-Ponty (2002), a fenomenologia é o estudo da essência. Por exemplo, a essência da percepção ou a essência da consciência. Em termos de pesquisa em turismo, a fenomenologia poderia ser aplicada na resolução de problemas que questionassem "como é ser um (a)...", no sentido de captar a essência da experiência de ser empreendedor no setor turístico, ou chefe executivo de um grupo hoteleiro, ou turista.

\footnotetext{
${ }^{2}$ HUSSERL, E. A idéia da fenomenologia. Lisboa: Edições 70, 1986 e HUSSERL, E. Investigações lógicas: sexta investigação: elementos de uma elucidação fenomenológica do conhecimento. Coleção Os Pensadores, São Paulo, Nova Cultural, 1988.
} 


\section{Grounded theory}

Além de sua denominação não ter equivalente na língua portuguesa, o entendimento do que é e a aplicabilidade da grounded theory nos estudos organizacionais e na pesquisa em turismo ainda gera discussões. Glaser e Strauss (1967) denominaram grounded theory como a maneira de se 'descobrir teoria a partir de dados' sistematicamente coletados da pesquisa social.

De acordo com Creswell (1998) para estudar como as pessoas agem e reagem a um determinado fenômeno, o pesquisador necessita de ampla gama de instrumentos de coleta de dados, em sua maioria primários, como informações de entrevistas e de visitas de campo, para desenvolver e inter-relacionar categorias de informações que os auxiliem as proposições teóricas/hipotéticas e que lhe apresentem uma visão da teoria.

Suddaby (2006) apresenta a existência de diversos conceitos distorcidos do que é grounded theory e quais suas características, como: a 'proibição' do pesquisador em ler sobre a teoria existente antes da coleta de dados, a confusão com os pressupostos da pesquisa fenomenológica, a utilização da grounded theory para testar hipóteses etc. $\mathrm{O}$ autor complementa que a grounded theory é um processo interpretativo que depende da sensibilidade do pesquisador em face dos elementos tácitos dos dados ou significados e conotações que podem não estar aparentes numa visão superficial do que ocorre na realidade.

Segundo Clark et al (1998) este método é mais comumente aplicado às pesquisas sobre hospitalidade, onde entrevistas em profundidade tem maior probabilidade de serem feitas e respondidas.

\section{Pesquisa-ação}

A dificuldade em alcançar o rigor científico, pois a objetividade consiste em limitar a parcialidade dos pontos de vista, é uma das características da pesquisa-ação. Thiollent (1997, p. 19) complementa que "no contexto da atuação sociopolítica, trata-se de uma pesquisa cuja finalidade consiste em esclarecer os objetivos, as implicações da ação ou as condições de mobilização requeridas para o êxito dessa ação". Avison et al 
(1999, p. 96) destacam que "na pesquisa-ação a ênfase é maior naquilo que os praticantes fazem, do que no que dizem fazer".

Argyris et al (1985) identificam que a ciência ação é uma investigação sobre como o homem delimita e implementa ação em relação ao outro, estando intimamente ligada à intervenção social. Para Avison et al (1999), a pesquisa-ação combina teoria e prática através da mudança e reflexão em situações problemáticas imediatas. Nesse sentido, Thiollent (1997) afirma que o maior objetivo da pesquisa é produzir novas informações, estruturar conhecimentos e delinear ações. Tem-se aqui a junção entre a prática diária e a teoria existente num processo de produção de conhecimentos e de intervenções na realidade dos atores sociais.

Algumas temáticas que caracterizam a pesquisa-ação foram compiladas de diversos autores por Argyris et al (1985, p. 8-9) e resultaram nas seguintes observações: (a) a pesquisa-ação envolve mudanças experimentais de problemas reais em sistemas sociais; (b) a pesquisa-ação envolve ciclos interativos de identificação de problemas, planejamento, ação e avaliação; (c) a mudança pretendida tipicamente envolve reeducação; (d) a pesquisa-ação desafia o statu quo a partir de uma perspectiva de valores democrática; (e) a pesquisa-ação pretende contribuir simultaneamente com o conhecimento básico nas ciências sociais e a ação social do dia-a-dia.

Ritchie e Goldner (1994) apresentam que, de maneira geral, a pesquisa-ação deve ter como objetivo entender o funcionamento de operações e programas com vistas a modificá-las. A presença do elemento prático, transformador, permeia toda a aplicação desta técnica.

\section{Observação participante}

A utilização da observação participante como meio de adentrar o mundo social do indivíduo já é de grande conhecimento de antropólogos e sociólogos. Nos estudos organizacionais, sua importância é crescente; uma das razões explicativas é a questão de não haver outra forma de o pesquisador conseguir acesso a determinado campo.

Outra consideração do método recai sobre a possibilidade de o pesquisador aprender mais sobre as atividades das pessoas em seu contexto natural por meio de 
observação e participação nessas atividades (KAWULICH, 2005); a possibilidade de o pesquisador compartilhar experiências não somente observando o que está acontecendo, mas também sentindo (SAUNDERS, LEWIS e THORNHILL, 2000).

Com relação ao futuro da observação participante, Adler e Adler (1994) postulam que é improvável que o método desapareça na medida em que se torne aclamado pelos pesquisadores; entretanto é provável que se difunda o uso integrado a outros métodos e técnicas de pesquisa.

De maneira geral e conforme apresentadas na tabela 02, as estratégias qualitativas de pesquisa apresentam diferentes mecanismos para se estudar um fenômeno. Entretanto todas as técnicas apresentadas têm em comum o objetivo de qualquer pesquisa qualitativa, conforme destacam Ritchie e Goeldner:

Com os tipos de pesquisa qualitativa o objetivo é desenvolver importantes mas limitadas - informações de cada indivíduo e falar com um número determinado de indivíduos para realizar as inferências sobre a população como um todo (RITCHIE e GOELDENER, 1994, p. 487).

\section{TABELA 02 - COMPARATIVO ENTRE AS ESTRATÉGIAS DE INVESTIGAÇÃO NA PESQUISA QUALITATIVA}

\begin{tabular}{|c|c|c|c|c|}
\hline Estratégia & Aplicações & Autores & Pontos fortes & “Preconceitos"/Limitações \\
\hline $\begin{array}{l}\text { Estudo de } \\
\text { caso }\end{array}$ & $\begin{array}{l}\text { Estudo de acontecimentos } \\
\text { contemporâneos, resposta } \\
\text { a questões do tipo 'como' } \\
\text { e 'por que' (YIN, 2001). } \\
\text { Testar teoria, produzir } \\
\text { descrições e gerar teoria } \\
\text { (EISENHARDT, 1989) }\end{array}$ & $\begin{array}{l}\text { Yin (2001, } \\
\text { 2003) } \\
\text { Stake (1995) } \\
\text { Gomm, } \\
\text { Hammersley } \\
\text { e Foster } \\
\text { (2000) }\end{array}$ & $\begin{array}{l}\text { Múltiplas fontes de } \\
\text { evidência (YIN, 2001). } \\
\text { Informações detalhadas } \\
\text { sobre o caso (GOMM e } \\
\text { HAMMERSLEY, 2000) } \\
\text { Emprego de múltiplos } \\
\text { níveis de análise (YIN, } \\
\text { 2001). }\end{array}$ & $\begin{array}{l}\text { Critérios de escolha dos casos e } \\
\text { processo de generalização } \\
\text { (STAKE, 2000) } \\
\text { Tempo para realização e } \\
\text { inúmeros documentos ilegíveis } \\
\text { (YIN, 2001) }\end{array}$ \\
\hline Etnografia & $\begin{array}{l}\text { Entendimento do } \\
\text { significado do trabalho e } \\
\text { alienação, estudos sobre } \\
\text { relações de trabalhadores } \\
\text { (HODSON, 1998) } \\
\text { Estudo do comportamento, } \\
\text { linguagem e interações de } \\
\text { uma cultura compartilhada } \\
\text { (CRESWELL, 1998) }\end{array}$ & $\begin{array}{c}\text { Wolcott } \\
(1994) \\
\text { Hammersley } \\
\text { e Atkinson } \\
\text { (1995) }\end{array}$ & $\begin{array}{l}\text { Ser uma forma básica de } \\
\text { pesquisa social } \\
\text { (HAMMERSLEY e } \\
\text { ATKINSON, 1995) } \\
\text { Descrições em um alto } \\
\text { nível de detalhes } \\
\text { (CRESWELL, 1998) }\end{array}$ & $\begin{array}{l}\text { Ser guiada tanto pelo teor } \\
\text { quanto pela delimitação da } \\
\text { pesquisa (VAN MAANEN, } \\
\text { 1979) } \\
\text { Tempo extensivo dedicado à } \\
\text { coleta de dados, possibilidade } \\
\text { do pesquisador 'se tornar um } \\
\text { nativo' e não completar seu } \\
\text { estudo (CRESWELL, 1998) }\end{array}$ \\
\hline
\end{tabular}


Continuação ...

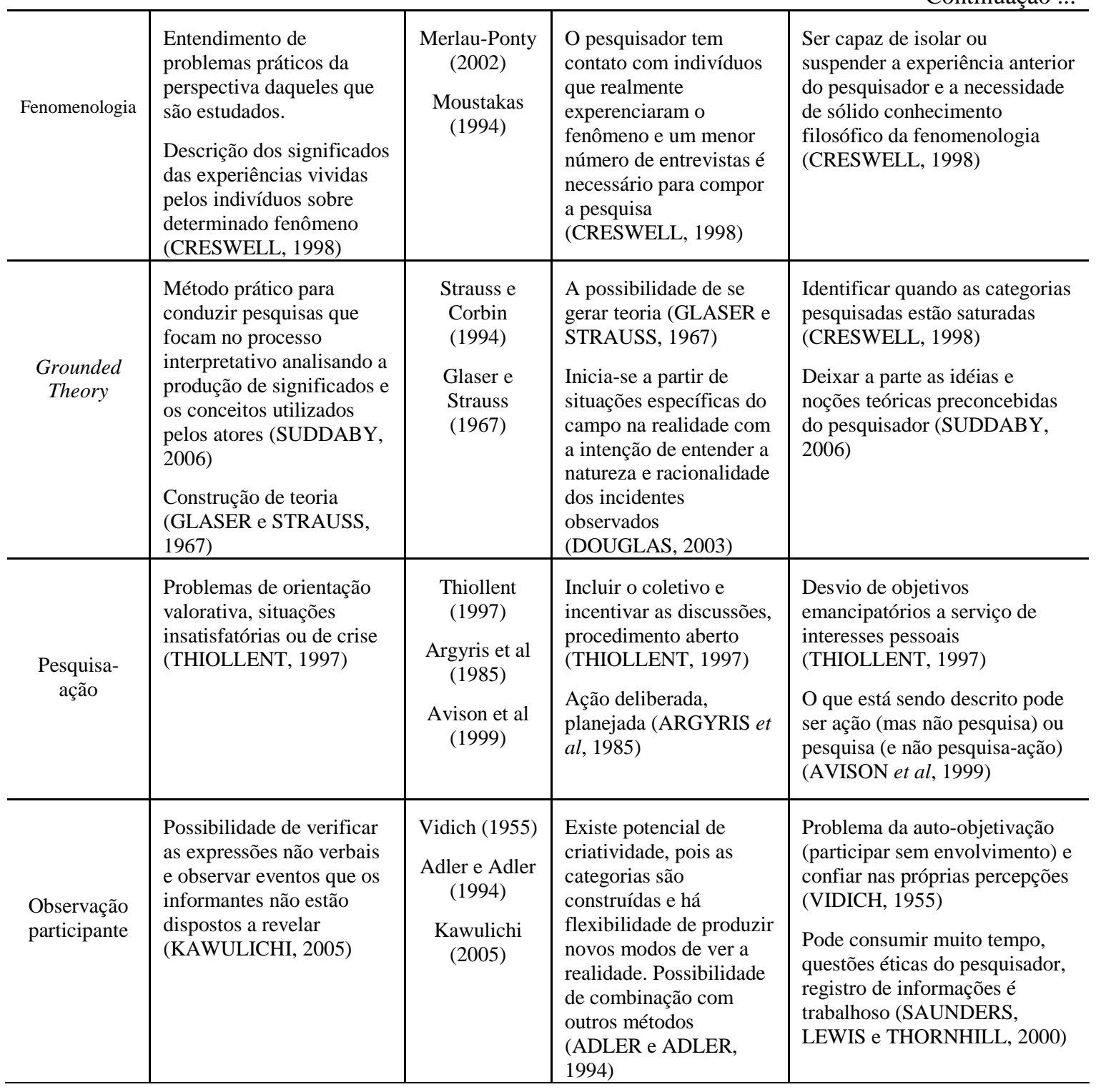

FONTE: Elaborado pela autora

Em face da diversidade de estratégias de investigação existentes e do emprego de diversos instrumentos na coleta de dados, o pesquisador poderia, conforme Miles e Huberman (1994), se deparar com alguns pares irreconciliáveis.

Dessa maneira, na tentativa de aproximar as estratégias e definir práticas analíticas comuns à pesquisa qualitativa como um todo, é interessante destacar as três etapas descritas por Wolcott (1994) como uma modalidade de uniformização do processo de pesquisa qualitativa. 
A descrição, segundo o autor, é a essência da investigação qualitativa e requer a distinção entre 'o que observar e anotar' e 'o que não anotar', 'o que é foco' e 'o que é periférico', enfatizando o aspecto crítico do trabalho descritivo. Na segunda etapa, a análise é apresentada como "aquilo que se faz no processo de cuidadosamente teorizar por meio dos dados" (WOLCOTT, 1994, p. 174), "mostrando como as coisas funcionam, identificando fatores chaves e relações" (MILES e HUBERMAN, 1994, p. $14)$.

O último elemento é a interpretação ou a dimensão interpretativa que representa o esforço de ir além das fronteiras de um caso particular, à procura de uma aplicação ou significado mais amplo dos dados. Wolcott (1994, p. 256) destaca que a interpretação são as repostas para o questionamento inerente a qualquer um que conduza uma pesquisa qualitativa e que queira descobrir o seu propósito ou em outras palavras: “e daí?”.

\section{CONSIDERAÇÕES FINAIS E DISCUSSÕES}

Como observado anteriormente, além da conceituação do que é pesquisa qualitativa ser conflitante, as múltiplas estratégias de investigação também ratificam a dificuldade de se delinear e conduzir uma pesquisa qualitativa.

A complexidade dessa abordagem, evidenciada em seu processo de pesquisa, não pode ser solucionada apenas com a escolha de uma tradição de investigação. Patton (2002, p. 134) sugere, de maneira espirituosa, que a distinção entre as estratégias de pesquisa deve ser feita respondendo-se a seis perguntas referentes à ontologia, epistemologia, metodologia, filosofia, disciplinaridade/interdisciplinaridade e o debate da prática da pesquisa. Nas palavras do autor "uma para cada dia da semana, deixando um dia para integrar as respostas".

Partindo-se da idéia assinalada por Denzin e Lincoln (2006, p. 36) de que "uma estratégia de investigação também compreende habilidades, suposições e práticas que o pesquisador emprega ao deslocar-se do paradigma para o mundo empírico" deve-se 
ressaltar que o sucesso da utilização de determinada estratégia tem como componente fundamental o pesquisador.

Dessa forma, a aplicação de quaisquer estratégias de investigação aos estudos e pesquisa em turismo e hospitalidade sem que o pesquisador considere seus pressupostos filosóficos, bem como o preparo para a utilização de tais métodos e técnicas pode incorrer na problemática da incoerência e inconsistência entre dados e realidade, invalidando a pesquisa ou boa parte dela.

Ritchie e Goeldner (1994) afirmam que a pesquisa qualitativa em turismo geralmente é utilizada para gerar hipóteses que sugiram soluções para as demandas e dificuldades das organizações.

Outra questão necessária ao processo de investigação qualitativa é a delimitação do estudo. Embora a estratégia de pesquisa escolhida guie o processo de coleta e análise dos dados, não se pode somente emprestar a lógica de tal estratégia e aplicá-la diretamente ao estudo. Maxwell (2005) aponta que a delimitação na pesquisa qualitativa é um processo contínuo.

Independentemente das estratégias de investigação escolhidas, conforme ressaltam Miles e Huberman (1994), o desafio para todos os pesquisadores qualitativos é descobrir descrições coerentes e explicações que ainda incluam todos os gaps, inconsistências e contradições inerentes à vida social e pessoal. A triangulação ou mensuração múltipla (BREWER e HUNTER, 2006), em que o pesquisador aplica diferentes técnicas de pesquisa para observar seu objeto de estudo, pode auxiliar a distinguir de forma acurada tais descobertas.

Da mesma forma Saunders et al (2000) suscitam a importância da revisão de literatura como uma atividade que antecede a pesquisa de campo, mas que continua, à medida que o estudo evolui, podendo ser visto como uma espiral.

Logo, Avison et al (1999) atentam que uma vantagem dos métodos qualitativos é o seu valor em explicar o que está acontecendo nas organizações. Finalmente, corroborando tal perspectiva, Rosen (1991, p. 21, grifo nosso) afirma que a razão para se escolher uma abordagem metodológica é uma questão de escolha estética, "envolvida mais com o que o pesquisador decide estudar e com o como ele fará isso". 


\section{REFERÊNCIAS BIBLIOGRÁFICAS}

ADLER, P. A.; ADLER, P. Observational tecniques. In: DENZIN, N. K.; LINCOLN, Y. S. Handbook of qualitative research. California: Sage Publications, 1994.

ARGYRIS, C.; PUTNAM, R.; SMITH, D. M. Action science: concepts, methods and skills for research and intervention. New York: Jossey Bass, 1985. Disponível em: http://www.actiondesign.com/action_science. Acesso em: 29/05/2007.

AVISON, D.; LAU, F.; MYERS, M.; NIELSEN P. A. Action research. Communications of the ACM, jan. 1999, v. 42, n. 1. Disponível em: http://www.cs.vu.nl/ gordijn/QRM/lib/CACM-Action\%20Research-AvisonJan1999.pdf. Acesso em: 01/08/2007

BORMAN, K. M.; LeCOMPTE, M. D; GOETZ, J. P. Ethnographic and qualitative research design and why it doesn't work. The American Behavioral Scientist. Sep/Oct 1986, 30, 1, p. 42-57.

BREWER, J.; HUNTER, A. Foundations of multimethod research: synthesizing styles. California: Sage Publications, 2006.

BRYMAN, A. The debate between quantitative and qualitative research: a question of method or epistemology? The British Journal of Sociology. Mar 1984, 35, 1, p. 75-92.

CASSELL, C.; SYMON, G. Qualitative methods in organizational research: a practical guide. London: Sage Publications, 1994.

CLARK, M. A.; RILEY, M. J.; WILKIE, E.; WOOD, R. Researching and writing dissertations in hospitality and tourism. London: International Thomson Business Press, 1998.

CRESWELL, J. W. Qualitative inquiry and research design: choosing among five traditions. Thousand Oaks, California: Sage Publications, 1998.

Research design: qualitative, quantitative and mixed method approaches. Thousand Oaks, California: Sage, 2003. 
DENZIN, N. K.; LINCOLN, Y. S. (org) O planejamento da pesquisa qualitativa: teorias e abordagens. Porto Alegre: Artmed, 2006.

DOUGLAS, D. Grounded theories of management: a methodological review. Management Research News. v. 26, n. 5, 2003, p. 44-52.

EISENHARDT, K. M. Building theories from case study research. Academy of Management Review. v. 14, n. 4, 1989, p. 532-550.

GLASER, B.; STRAUSS, A. The Discovery of Grounded Theory: strategies for qualitative research. Chicago: Aldine, 1967.

GOMM, R.; HAMMERSLEY, M.; FOSTER, P. Case study methods: key issues, key texts. London: Sage Publications, 2000.

GRIX, J. Introducing studentes to the generic terminology of social research. Politics. 2002, 22, 3, p. 175-186.

HAMMERSLEY, M.; ATKINSON, P. Ethnography: principles in practice. New York: Routledge, 1995.

HODSON, R. Organizational ethnographies: an underutilized resource in the sociology of work. Social Forces. v. 76, n. 4, jun. 1998, p. 1173-1208.

KAWULICH, B. B. Participant observation as a data collection method. Forum: Qualitative Social Research [On-line journal]. v. 6, n. 2, art. 43. 2005. Disponível em: http://www.qualitative-research.net/fqs-texte/2-05/05-02-43-e.htm. Acesso em: 23/05/07

LYNCH, P. A. Networking in the homestay sector. The Service Industries Journal. v. 20, n. 3 , jul. 2000.

MARSH, D.; FURLONG, P. A Skin not a Sweater: Ontology and Epistemology in Political Science. In: MARSH, D.; STOKER, G. (eds). Theory and Methods in Political Science Palgrave: Basingstoke, 2002, p. 17-44.

MAXWELL, J. A. Qualitative research design: an integrative approach. Applied Social Research Methods Series. v. 41. London: Sage Publications, 2005. 
MERLAU-PONTY, M. Phenomenology of perception. London, UK: Routledge, 2002.

MILES, M.B.; HUBERMAN, A. M. Qualitative data analysis: an expanded sourcebook California: Sage, 1994.

MOUSTAKAS, C. Phenomenological research methods. Thousand Oaks: Sage, 1994.

NOVELLI, M.; SCHMITZ, B. SPENCER, T. Networks, clusters and innovation in tourism: a UK experience. Tourism Management. v. 27, 2006.

PATTON, M. Q. Qualitative research and evaluation methods. California: Sage, 2002.

PUNCH, K. F. Introduction to social research: quantitative and qualitative approaches. London: Sage, 1998.

RITCHIE, J. R. B.; GOELDNER, C. R. Travel, tourism, and hospitality research: a handbook for managers and researchers. New York: John Wiley \& Sons, 1994.

ROSEN, M. Coming to terms with the field: understanding and doing organizational ethnography. Journal of Management Studies. v. 28, n. 1, jan. 1991, p. 1-24.

SANDAY, P. R. The ethnographic paradigm(s). Administrative Science Quarterly, v. 24, 1979, p. 527-538.

SAUNDERS, M. N. K.; LEWIS, P.; THORNHILL, A. Research methods for business students. England: Pearson Education, 2000.

SAXENA, G. Relationships, networks and the learning regions: case evidence from the Peak District National Park. Tourism Management. v. 26, 2005.

SILVERMAN, D. Doing qualitative research: a practical handbook. London: Sage, 2000 .

STAKE, R. E. The art of case study research. Thousand Oaks, CA: Sage, 1995. 
The case study method in social inquiry. In: GOMM, R.; HAMMERSLEY, M.; FOSTER, P. Case study methods: key issues, key texts. London: Sage Publications, 2000 .

STRAUSS, A.; CORBIN, J. Grounded theory methodology: an overview. In: DENZIN, N.K.; LINCON, Y.S. Handbook of qualitative research. Beverly Hills, CA: Sage Publications, 1994.

SUDDABY, R. From the editors: what grounded theory is not. Academy of Management Journal. v. 49, n. 4, ago. 2006, p. 633-642.

THIOLLENT, M. Pesquisa-ação nas organizações. São Paulo: Ed. Atlas, 1997.

VAN MAANEN, J. The fact of fiction in organizational ethnography. Administrative Science Quarterly, v. 24, 1979, p. 539-550.

VIDICH, A. Participant observation and the collection and interpretation of data. The American Journal of Sociology. v. 60, n. 4, p. 354-360, 1955.

WOLCOTT, H. F. Transforming qualitative data: Description, analysis, and interpretation. Thousand Oaks, CA: Sage, 1994.

YIN, R. K. Applications of case study research. 2. ed. Thousand Oaks: Sage Publications, 2003.

Estudos de Caso: planejamento e métodos. Porto Alegre: Bookman , 2001.

Recebido em: 14 de setembro de 2007

Aprovado em: 14 de outubro de 2007 